\title{
Hemodynamic Changes and Compensatory Mechanisms during Early Cardiogenesis after Neural Crest Ablation in Chick Embryos
}

\author{
LINDA LEATHERBURY, DAVID M. CONNUCK, HAROLD E. GAULDIN, AND \\ MARGARET L. KIRBY \\ Section of Pediatric Cardiology [L.L., D.M.C., M.L.K.] and Department of Anatomy [D.M.C., H.E.G., M.L.K.], \\ Medical College of Georgia, Augusta, Georgia 30912
}

\begin{abstract}
Microcinephotography was used to study early heart development in chick embryos with ablations of the neural crest known to result in persistent truncus arteriosus with associated aortic arch anomalies. The premigratory neural crest destined for the 3rd and 4th pharyngeal arches and the aorticopulmonary septum were ablated. When the embryos reached the looped cardiac tube stage (stage 18), 15 experimental and 15 control embryos were filmed at 100 frames/s under controlled environmental conditions. End-diastolic and end-systolic dimensions were determined for the conotruncus and presumptive right ventricle that together compose the bulbus cordis. The results showed that the shortening fractions and ejection fractions were significantly depressed in the experimental embryos. The experimental embryos exhibited dilation and decreased emptying of the ventricle. There was no difference in heart rate or stroke volume between the control and experimental embryos. Thus, the calculated cardiac output was the same in the control and experimental groups. It appeared that the experimental embryos compensated for decreased contractility by ventricular dilation. These functional compensations in very early cardiac development may play an etiologic role in the subsequent development of structural heart defects. (Pediatr Res 30: 509-512, 1991)
\end{abstract}

The neural crest model of abnormal heart development in chick embryos (1) potentiates the study of the interrelationship of the functional and morphologic components of cardiogenesis. Ablation of premigratory neural crest destined for the septa of the cardiac outflow tract via the $3 \mathrm{rd}$ and 4 th pharyngeal arches leads to a $95 \%$ cardiovascular anomaly rate at d 11 of incubation. The cardiovascular structures affected include the outflow tract, inflow tract, and aortic arch arteries (2). Nishibatake et al. (2) showed that the majority of embryos had persistent truncus arteriosus with a variety of aortic arch artery anomalies that included interruption of the definitive aorta. This association of persistent truncus arteriosus with interruption of the aorta is also found in humans and has been proposed to be due to abnormal development of the neural crest (3).

Microcinephotography of the cardiovascular system on $\mathrm{d} 3$ of development showed abnormal function and morphologic characteristics of the hearts and aortic arch arteries in embryos with cardiac neural crest ablations (4). By visual analysis, there ap-

Received March 4, 1991; accepted July 23, 1991

Correspondence: Linda Leatherbury, M.D., Section of Pediatric Cardiology, BAA 8302, Medical College of Georgia, Augusta, GA 30912.

Supported by PHS Grant HL 36059 . peared to be depressed contractility and dilation of the ventricle, interruption of the 4th aortic arch artery, aortic sac incompetency, and incomplete looping of the cardiac tube. These abnormal characteristics are evidence of functional and morphologic changes that occur before the time in development when endpoint structural heart defects would be present. The current study was designed to assess, quantitatively, the contractility of the ventricle, dilation of the components of the developing heart, and cardiac output.

\section{MATERIALS AND METHODS}

Neural crest ablation. Fertilized Arbor Acre chicken eggs (Seaboard Hatchery, Athens, GA) were incubated in a forced-draft incubator at $37^{\circ} \mathrm{C}$ and $97 \%$ relative humidity. After $25-30 \mathrm{~h}$ of incubation, they were windowed and prepared for microsurgery as reported by Narayanan (5). The stage of development of the embryos was determined according to the stage development procedure of Hamburger and Hamilton (6). Each embryo was stained with neutral red, and the overlying vitelline membrane was torn. Surgical ablation by microcautery was performed on the embryonic neural fold at stages 8-10. In these experiments, neural fold was ablated bilaterally between the midotic placode and somite 2 . This region corresponds to the cardiac neural crest destined for pharyngeal arches 3 and 4 . A recent study by Tomita et al. (7) has shown that this particular neural crest lesion results in an $83 \%$ incidence of persistent truncus arteriosus and a $17 \%$ incidence of double outlet right ventricle, associated with a $100 \%$ incidence of aortic arch artery anomalies. After microsurgery, the eggs were sealed with cellophane tape and reincubated in the same high humidity incubator. Twenty-four $\mathrm{h}$ after surgery, they were transferred to a second incubator at $37^{\circ} \mathrm{C}$ and $70 \%$ relative humidity. Control eggs were incubated and transferred in parallel with experimental eggs. To allow for temperature reequilibration, the control embryos were windowed $30 \mathrm{~min}$ before microcinephotography.

Microcinephotography. Each egg was transferred to a heated sand bath. Using a thermistor probe placed on the surface of the yolk sac adjacent to the embryo, the temperature of the sand bath was adjusted so that the embryo was maintained at a constant temperature of $37.5^{\circ} \mathrm{C}$. The embryos were viewed $48 \mathrm{~h}$ after microsurgery and were filmed when they reached stage 18 . At this stage of development, the heart is a looped cardiac tube with a well-defined outflow tract and bilaterally present aortic arch arteries 2, 3, and 4. At stage 18, the embryo lies on the yolk sac such that right-sided cardiovascular structures are delineated by red blood cells that are seen through the transparent embryonic tissue. Limb buds do not obscure the cardiac tube or aortic arch arteries until later in development.

Fifteen control and 15 experimental embryos at stage 18 of development were selected for microcinephotography as previ- 
ously described by Leatherbury et al. (4). A power analysis indicated that 13 embryos per group were needed to have an $80 \%$ probability of obtaining statistically significant results at $\alpha$ $=0.05$ for an effect size of 1.0. Embryos with axial torsion that altered or obscured the visual characteristics of the cardiovascular system were not filmed. Experimental embryos that were selected for filming compared with the group that was not selected were shown in our laboratory to have the same survival rate, incidence of persistent truncus arteriosus, and abnormal aortic arch arteries (7). Filming was done with a high speed camera (Redlake Locam II; Redlake Corp., Morgan Hill, CA) mounted on a stereomicroscope (Olympus Corporation of America, New Hyde Park, NY) with lighting provided by two fiberoptic light sources. The primary light source was focused through a lens system attached to a strobe (Strobex model 236; Chadwick Helmuth, Elmonte, CA) that was externally triggered by the camera's shutter opening. The second light source was continuous. Each embryo was filmed for $5 \mathrm{~s}$ at a film rate of 100 frames/s, which resulted in 40 frames/ cardiac cycle for a typical embryo. Each egg was positioned in the sand bath at the same distance from the microscope lens, yielding a magnification factor of approximately $30 \times$ on the film (Kodak Ektachrome High Speed Daylight Film no. 7251). The embryo was filmed with a stage micrometer to allow exact calibration of the magnification factor.

Film analysis. The film was analyzed independently by two investigators who were not cognizant of the experimental status of the embryos. Interrater reliability was judged to be high with Pearson correlation coefficients in the range of $0.94-0.98$ on the dependent variables. The intrarater test-retest variability was $3 \%$ with the resolution of the digitizing pad at $25 \mu \mathrm{m}$. The analysis was performed using a projector specifically designed for highspeed cinephotographic film. Single frames were selected visually to represent end-systolic and end-diastolic points in the cardiac cycle. The end-diastolic frame was selected as the frame with the largest amount of red blood cells in the ventricle. The exact number of film frames counted between an end-diastolic frame and the subsequent end-systolic frame and the film speed of 100 frames/s were used to calculate the heart rate of each embryo.

At this stage of development, the transparent embryonic tissue allows the endocardial boundaries of the developing cardiovascular system to be delineated by the presence of intravascular red blood cells. Linear measurements and area determinations were obtained using a digitizing pad (9874A; Hewlett-Packard Co., Palo Alto, CA) and computer software programs specifically designed for electronic planimetry (8). Measurements were made on end-diastolic and end-systolic frames for three cardiac cycles per embryo (Fig. 1). The width and length of the two component regions of the bulbus cordis, the conotruncus and ventricle, were determined. The areas of three regions were measured: the ventricle, conotruncus, and bulbus cordis. Volume determinations were calculated based on a modification of the two-chamber method for determining right ventricular volumes in children (9). From microscopic visualization of the embryos and scanning electron micrographs (10), it was determined that the conotruncus approximated the shape of a cylinder and the ventricle approximated the shape of a prolated ellipsoid. The assumption was made that the rotation of the areas of the conotruncus and the ventricle on their long axes would yield a more accurate estimation of volumes than the basic two-chamber method. The traced areas of the ventricle and conotruncus were rotated to complete $360^{\circ}$ on their long axes, and the area or volume of the ventricle and the conotruncus were summed to yield the area or volume of the bulbus cordis. Analyses of real-time microcinephotography (4) and pressure-volume loops in normal chick embryos have shown that there is egress of red blood cells into the conotruncus during diastolic filling of the ventricle at early stages of cardiovascular development (11). Thus, the bulbus cordis was considered to be the most appropriate single structural unit whose stroke volume would best determine cardiac output at this point in early development.

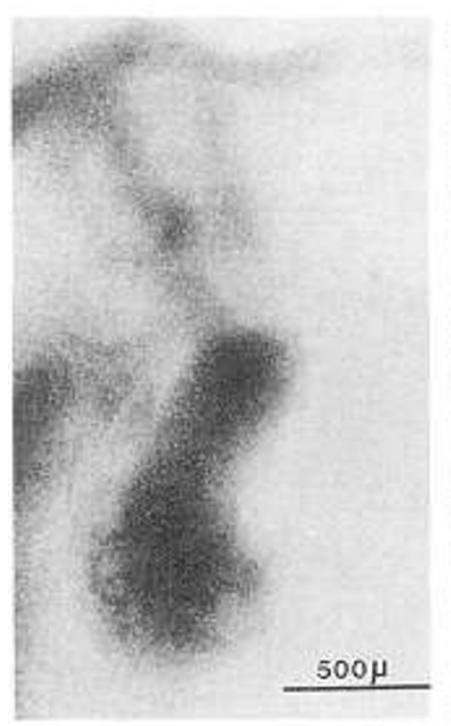

Control End-diastole
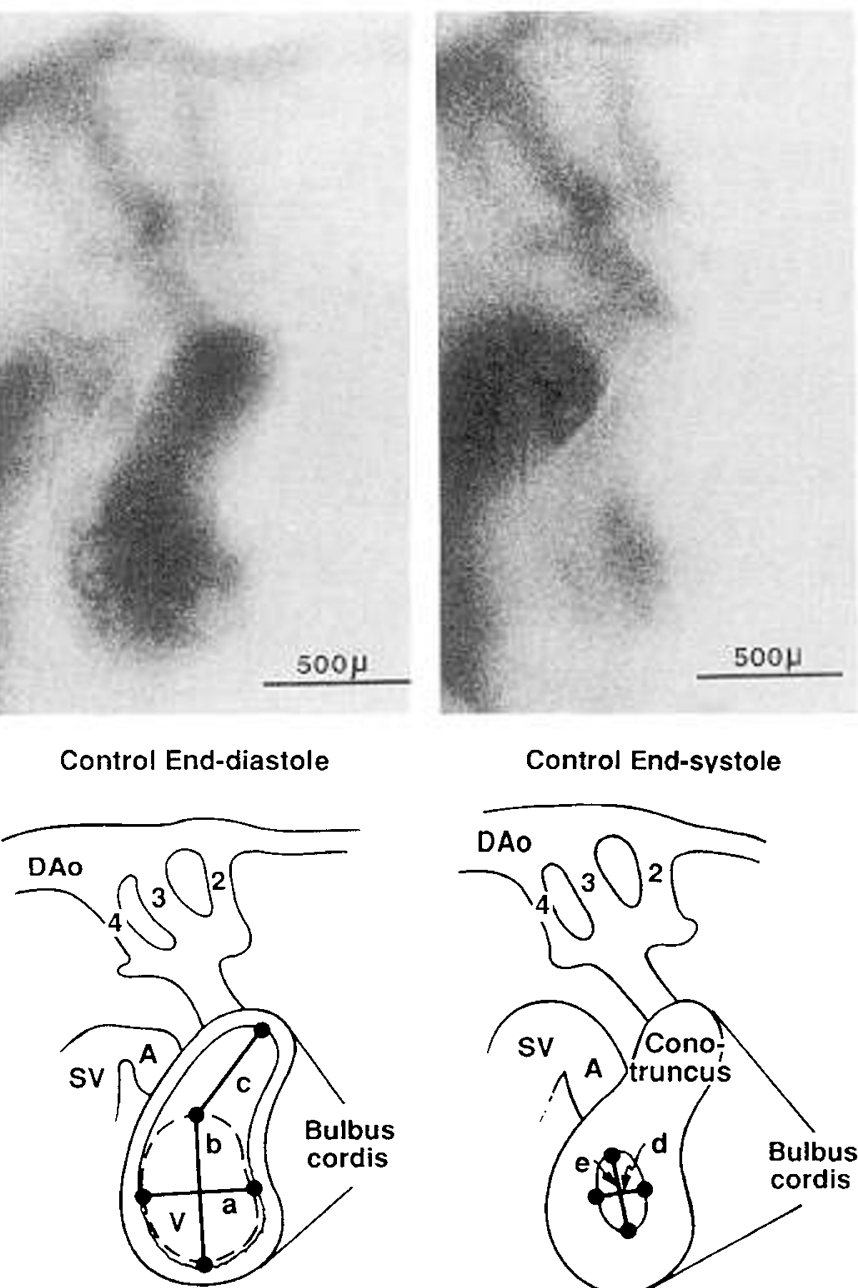

Control End-systole

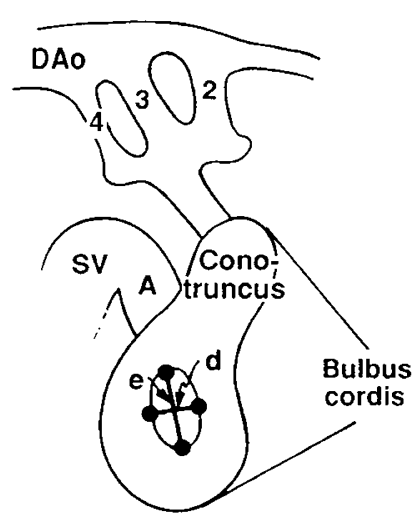

Fig. 1. Photomicrograph and line drawing composites of a stage 18 control embryo to exemplify end-diastole measurements. $A$, atrium; $D A O$, dorsal aorta; $V$, ventricle; 2 , right 2 nd aortic arch artery; 3 , right 3 rd aortic arch artery; 4 , right 4 th aortic arch artery; $S V$, sinus venosus; $a$, ventricular end-diastolic diameter; $b$, ventricular end-diastolic length; $c$, conotruncal diastolic length; $d$, ventricular end-systolic diameter; $e$, ventricular end-systolic length.

Several indices of contractility were calculated. The shortening fraction of the primitive ventricle was calculated using the middiameter of the ventricle in end-diastole and end-systole. Ejection fractions for both the bulbus cordis and the ventricle were calculated using area and volume dimensions. The change in area of the bulbus cordis between end-diastole and end-systole as well as the stroke volume were multiplied by the heart rate to yield cardiac flow in area $\left(\mathrm{mm}^{2} / \mathrm{min}\right)$ and cardiac output in volume $\left(\mathrm{mm}^{3} / \mathrm{min}\right)$, respectively. These parameters were compared for control and experimental embryos using analysis of variance with the level of statistical significance chosen as a $p$ value of less than 0.05 .

\section{RESULTS}

Previous visual analysis of real-time microcinephotographic films showed dilation of the ventricle and decreased emptying of the bulbus cordis (4). Comparing the same developmental stage of embryos, the results of this quantitative study produced area and volume measurements of the total bulbus cordis and its components, the conotruncus and ventricle. The results, as seen in Table 1, show that the bulbus cordis in end-diastole and endsystole in the experimental embryos is significantly larger than the same structure in control embryos. Further analysis of the 
Table 1. Bulbus cordis areas and volumes-stage 18 (mean \pm SD)

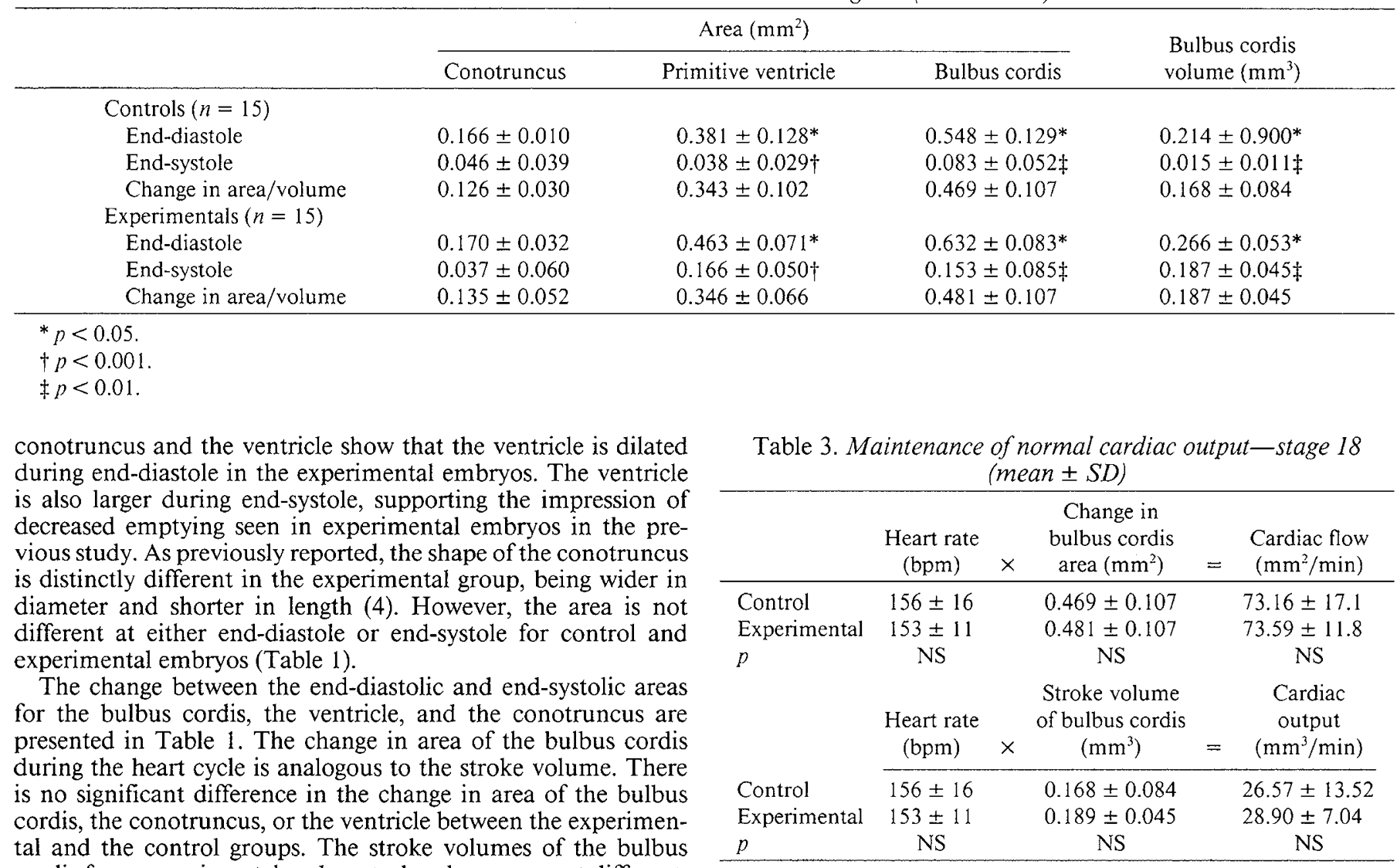
cordis from experimental and control embryos are not different, with the bulbus cordis of the experimental embryos appearing dilated in both diastole and systole.

Table 2 shows the shortening and ejection fractions, which are load-dependent indices of contractility. The experimental group has a significantly depressed shortening fraction $(53 \%)$ in comparison to the control group $(76 \%)$. Derivative calculations from the one-dimensional measurement of shortening fraction to the two-dimensional measurement of percentage change in area and the three-dimensional measurement of percentage of change in volume produce similar relationships between groups. The ejection fractions are significantly lower in the experimental embryos than in the control embryos. The assumptions involved in these derivative calculations do not appear to alter the significance of these relationships between the experimental and control groups. These quantitative indices of contractility support the qualitative assessment (4) of depressed contractility of the ventricle in the experimental embryos.

It is important to assess whether the cardiac output is similar between the control and the experimental embryos because contractility is depressed. As seen in Table 3, there is no significant difference in heart rate between the control and experimental embryos. Neither the change in area of the bulbus cordis nor the stroke volume of the bulbus cordis is decreased in the experimental group. Cardiac flow $\left(\mathrm{mm}^{2} / \mathrm{min}\right)$ and cardiac output

Table 2. Indices of contractility (mean $\pm S D$ )

\begin{tabular}{lccc}
\hline & Control & Experimental & $p$ \\
\hline $\begin{array}{l}\text { Shortening fraction (\%) } \\
\text { Ejection fraction }\end{array}$ & $76 \pm 8$ & $53 \pm 12$ & $<0.0001$ \\
Bulbus cordis & & & \\
$\quad$ Area \% change & $86 \pm 3$ & $76 \pm 8$ & $<0.0001$ \\
$\quad$ Volume \% change & $97 \pm 2$ & $87 \pm 8$ & $<0.0001$ \\
Primitive ventricle & & & \\
Area \% change & $90 \pm 2$ & $75 \pm 8$ & $<0.0001$ \\
Volume \% change & $97 \pm 2$ & $87 \pm 8$ & $<0.0001$ \\
\hline
\end{tabular}

$\left(\mathrm{mm}^{3} / \mathrm{min}\right)$ are not significantly different between the experimental and control groups.

\section{DISCUSSION}

Qualitative analysis of microcinephotography films of early development of the cardiovascular system showed significant differences between embryos with neural crest ablations and control embryos (4). The experimental embryos had dilated ventricles with decreased emptying and depressed contractility. The present study is a quantitative assessment of these features at the same developmental stage. We have shown that the bulbus cordis in experimental embryos is dilated at end-diastole and end-systole. This dilation is most pronounced in the ventricular portion of the bulbus cordis. The shortening and ejection fractions, as indices of contractility, are significantly decreased in experimental embryos in comparison to control embryos. The experimental embryos do not appear to compensate for the decreased contractility with an increased heart rate or stroke volume. However, the cardiac output was similar between the control and experimental groups. It appears that the experimental embryos compensate for decreased contractility by ventricular dilation. This maintains a normal stroke volume and, therefore, a normal cardiac output. These results suggest that morphologic development of the heart is subservient to normal physiologic requirements during cardiac morphogenesis.

Altered hemodynamic variables and compensations for them occur before the time when structural defects described for the phenotypically mature cardiovascular system are present. We believe that these hemodynamic alterations may be an additional factor in the phenotypic expression of structural heart defects.

The microscopic size of the developing cardiovascular system imposes technical limitations on functional measurements. The measurements of contractility used in this study are loaddependent indices. It is possible that these indices of contractility may be operating under different loaded conditions between the 
control and the experimental groups. This model of neural crest ablation has been shown to result in $100 \%$ of embryos with anomalies of the derivatives of the aortic arch arteries (7). Fifty percent of these embryos have interrupted aortic arch, and the other anomalies are less severe. We reported previously that $50 \%$ of the experimental embryos at stage 18 had either severely decreased blood flow or absence of blood flow in the right 4th aortic arch, which becomes the definitive aorta (4). These early alterations in blood flow through the aortic arch arteries provide a potential source of increased afterload to the developing heart in the experimental embryos. Thus, an alternate explanation for the decrease in shortening and ejection fractions is that it is a response to increased afterload.

Although a previous study showed that there is a substantial peak systolic pressure gradient between the ventricle and the dorsal aorta, this gradient is seen in both control and experimental embryos (12). This is substantiated by the fact that the cardiac output is similar in both the control and experimental groups, indicating that there is no evidence of increased resistance to blood flow across the total sum of aortic arch arteries at this stage of development. Thus, there is no empirical evidence for an increased afterload causing the decreased shortening and ejection fractions.

Other etiologies for the ventricular dilation in the experimental embryos need to be considered. Our previous study described blood returning to the midpoint of the conotruncus from the aortic sac (4). However, blood was never seen to regurgitate into the proximal conotruncus or into the primitive right ventricle. Even in the group with the most severe backflow of blood, the most proximal part of the conotruncal cushions were tightly sealed until the next bolus of blood was pushed through in late diastole. This regurgitation of blood was incorrectly described as incompetence of the truncal cushions, but is more appropriately referred to as aortic sac insufficiency. Thus, regurgitant blood is not presented to the ventricle and would not be a cause of ventricular dilation, nor would it increase the cardiac output.

The observations in a previous study that both circumferential and meridional wall stresses are significantly elevated in the neural crest-ablated experimental embryos support the notion that either a volume overload or ventricular dilation due to the myocardial dysfunction may be present (12). Investigations of neural crest-ablated embryos at the cellular level suggest that primary myocardial dysfunction does occur (13). Myocardial dysfunction in turn could explain the depressed indices of contractility.

In summary, this quantitative study supports our previous qualitative observations of cardiovascular changes in embryos with neural crest ablation. Before the heart is phenotypically mature, there are obvious morphologic abnormalities during very early cardiovascular development. This study shows that the experimental embryos have compensated physiologically for the insult to the cardiovascular system as early as the looped cardiac tube stage. In particular, these embryos with depressed contractility are compensating via the Frank-Starling relationship. The embryonic ventricle dilated to maintain a normal stroke volume for an adequate cardiac output. The Frank-Starling mechanism was also described by Wagman et al. (14) in response to volume loading of chick embryos at a similar stage of development. It is striking that the developing cardiovascular system, which is not innervated and is completely immature morphologically and cellularly, is able to exhibit compensatory mechanisms for embryonic survival.

Acknowledgments. The authors thank Karen Waldo for medical illustration, Harry Davis for statistical consultation, and Carol Pennington for manuscript preparation.

\section{REFERENCES}

1. Kirby ML, Waldo KL 1990 Role of neural crest in congenital heart disease. Circulation 82:332-340

2. Nishibatake M, Kirby ML, Van Mierop LHS 1987 Pathogenesis of persistent truncus arteriosus and dextroposed aorta in the chick embryo after neural crest ablation. Circulation 75:255-264

3. Van Mierop LHS, Kutsche LM 1986 Cardiovascular anomalies in DiGeorge syndrome and importance of neural crest as a possible pathogenic factor. Am J Cardiol 58:133-137

4. Leatherbury L, Gauldin HE, Waldo K, Kirby ML 1990 Microcinephotography of the developing heart in neural crest-ablated chick embryos. Circulation 81:1047-1057

5. Narayanan $\mathrm{CH} 1970$ Apparatus and current techniques in the preparation of avian embryos for microsurgery and for observing embryonic behavior. Biosciences 20:868-871

6. Hamburger V, Hamilton HL 1951 A series of normal stages in the development of the chick embryo. J Morphol 88:49-92

7. Tomita H, Connuck DM, Leatherbury L, Kirby ML 1991 Relation of early hemodynamic changes to final cardiac phenotype and survival after neural crest ablation in chick embryos. Circulation 84:1289-1295

8. Santi PA, Fryhofer J, Hansen G 1980 Electronic planimetry. Byte (March): 114 122

9. Graham Jr TP, Jarmakani JM, Atwood GF, Canent RV 1973 Right ventricular volume determinations in children: normal values and observation with volume or pressure overload. Circulation 67:144-153

10. Bockman DE, Redmond ME, Kirby ML 1990 Altered development of pharyngeal arch vessels after neural crest ablation. Ann NY Acad Sci 588:296304

11. Keller BB, Hu N, Serrino PJ, Clark EB 1989 Pressure-area loop characteristics in the stage 6 to 24 chick embryo. Circ Res 68:226-231

12. Leatherbury L, Braden DS, Tomita H, Gauldin HE, Jackson WF 1990 Hemodynamic changes: wall stresses and pressure gradients in neural crest-ablated chick embryos. Ann NY Acad Sci 588:305-313

13. Creazzo TL 1990 Reduced L-type calcium current in the embryonic chick heart with persistent truncus arteriosus. Circ Res 66:1491-1492

14. Wagman A, Hu N, Clark EB 1990 Effect of changes in circulating blood volume on cardiac output and arterial and ventricular blood pressure in the stage 18, 24, and 29 chick embryo. Circ Res 67:187-192 